\title{
Introduction to the Special Issue on Technical and Social
} Advances in HRI: An Invitational Issue of JHRI

\author{
Michael A. Goodrich \\ Brigham Young University
}

One of the goals of the Journal of Human-Robot Interaction (JHRI) is to promote a stronger research community, including interactions among HRI-relevant conferences and journals. This issue is comprised of papers that first appeared in another format, usually a conference, and that exhibited special insights or area of interest. Authors were invited to submit extensions to these papers with at least $50 \%$ new material and told that the papers would be reviewed relative to the standard of scholarship suited to an archival journal. Submitted papers were then reviewed by at least three reviewers, who were instructed to hold the papers to a journal-level standard for scholarship. Of the papers that were invited, five papers were selected for publication.

Invited papers were selected from two pools of papers: (1) previous submissions to special issues of JHRI that were interesting to reviewers but that had one or two technical defects that prevented them from being published and (2) papers that were wellreceived in HRI-related conferences. The conference papers were selected by contacting members of the program committee of a set of conferences and asking them to nominate especially interesting or noteworthy papers. The set of conferences that were considered were: HRI 2012, Ro-MAN 2011, RSS 2012, IROS 2012, ICRA 2012, and CHI 2012. There are other HRI-related conferences, but logistical considerations prevented considering papers from every HRI-related conference.

The first paper in this special issue is by Juan Fasola and Maja Matarić. This paper documents the systematic design and evaluation of a robot coach to help motivate the elderly to exercise. The results presented in the paper show that the systematic design approach produced a physical robot that was preferred by and motivated exercise better than a virtually embodied coach for two different age groups. Lessons in the paper generalize beyond the specific application area, having relevance for a broad set of social human-robot interactions.

The second paper is by Anca Dragan, Siddhartha Srinivasa, and Kenton Lee. This paper addresses the familiar problem of teleoperation in a novel way. The paper identifies commonalities across several forms of teleoperation, abstracts these commonalities into a function that retargets human input into robot behavior, and then evaluates how robot intelligence and customizable interfaces can be used to perform

\footnotetext{
Authors retain copyright and grant the Journal of Human-Robot Interaction right of first publication with the work simultaneously licensed under a Creative Commons Attribution License that allows others to share the work with an acknowledgement of the work's authorship and initial publication in this journal.
}

Journal of Human-Robot Interaction, Vol. 2, No. 2, 2013, Pages 1-2. DOI 10.5898/JHRI.2.2.Goodrich 
retargeting in a way that better supports human intent subject to some design principles distilled from careful evaluations.

The third paper is by Robin Deits, Stefanie Tellex, Pratiksha Thaker, Dimitar Simeonov, Thomas Kollar, and Nicholas Roy. This paper contributes to the rich literature on improving natural language for interactions between a human and a robot. The authors approach this problem as one of minimizing entropy in human-robot dialogue by having the robot ask clarifying questions. Results in the paper demonstrate important advantages of this approach over related approaches and also identify important limitations.

The fourth paper is by Chieng-Ming Huang and Bilge Mutlu. This paper describes the design of a set of robot social acts. Although the set of social acts is interesting of itself, the paper also supports these acts by proposing an activity model that maps these acts to an appropriate context for use and to an intended meaning. The paper further presents an implementation of these acts in the Robot Operating System (ROS) and an evaluation of the utility of the acts in various contexts.

The last paper is by Andreas Kolling, Katia Sycara, Steven Nunnally, and Michael Lewis. This paper addresses an emerging form of human-robot interaction, human-swarm interaction. The paper explores two fundamental types of interaction, intermittent and environmental, implements representative forms of these types in a testbed, and then evaluates the various implementations yielding observations on the scalability and usefulness of the two forms of human-swarm interaction.

Taken together, these papers demonstrate topical breadth in the field of HRI. Illustrating this breadth is important because there is a temptation among some members of the community to define HRI as applying only to one set of problems and solution approaches. The topics of these papers include applications and advances in probabilistic reasoning, teleoperation, system design, robot social interactions, and robot swarms. The papers collectively provide a sample of the various methods, from user studies to simulation results to field evaluations, by which results in HRI-related papers can be validated.

Many thanks go to the generous manuscript submitters, reviewers, and editors who made this special issue possible. We would like to thank especially Magnus Egerstedt and Andrea Thomaz for being special editors of two papers for which we either lacked the relevant knowledge to manage fairly or had a conflict of interest. We also appreciate the financial assistance or in-kind support given by various contributors to make the journal free for readers and authors; this support allows us to reach a global audience of the HRI community and beyond. We hope that these articles will be as insightful and useful for you as they are for us.

Managing editor's contact information: Michael A. Goodrich, Computer Science Department, Brigham Young University, Provo, Utah, USA. Email: mike@cs.byu.edu 